\title{
Motion Artifact Removal Techniques for Wearable Blood Pressure Measurement Device
}

\author{
Weiting Liu ${ }^{1}$, Fuyuan Xie ${ }^{1}$, Binpeng Zhan ${ }^{1}$ and Man $\mathrm{Xu}^{2 *}$ \\ ${ }^{1}$ State Key Laboratory of Fluid Power \& Mechatronic Systems, Zhejiang University, China \\ ${ }^{2}$ Public Sports and the Arts Department, Zhejiang University, China \\ *Corresponding author: Man Xu, Public Sports and the Arts Department, Zhejiang University, Hangzhou 310027, China. \\ To Cite This Article: Weiting Liu, Fuyuan Xie, Binpeng Zhan, Man Xu. Motion Artifact Removal Techniques for Wearable Blood Pressure \\ Measurement Device. Am J Biomed Sci \& Res. 2021 - 13(5). AJBSR.MS.ID.001911. DOI: 10.34297/AJBSR.2021.13.001911.
}

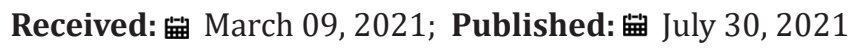

\begin{abstract}
With the development of mobile health technology, vital signs monitoring equipment based on wearable sensors is becoming a research focus. One of the key points of wearable vital signs detection technology is the removal of motion artifacts. Blood pressure is one important human physiological parameter, closely related to patients with hypertension, can be used as an important parameter to diagnose and prevent cardiovascular diseases. To improve the accuracy of the measurement of blood pressure by removing motion artifacts and then promote the wearable blood pressure measurement technology is very important. This article reviews the understanding of motion artifacts, motion artifact removal technology, and its research progress.
\end{abstract}

Keywords: Wearable Devices, Motion Artifact Removal, Digital Signal Processing

\section{Introduction}

According to the World Health Organization (WHO), cardiovascular diseases (CVDs) are the leading cause of death globally. An estimated 17.9 million people died from CVDs in 2019, representing $32 \%$ of all global deaths. It is estimated that the number of CVDs will continue to increase rapidly in the next decade, so the control and management of CVDs are of great significance.

Cardiovascular disease as a chronic disease, had become the main cause of global death and disability [1], the current main treatment strategy is prevention. As an important physiological parameter of the human body, blood pressure is one of the important indicators that reflect whether the cardiovascular function of the human body is good and can be used as an important parameter to diagnose and prevent cardiovascular diseases [2]. Therefore, daily blood pressure measurement is a very reliable and practical method for high-risk groups to understand the trend of blood pressure levels, and it is also an effective measure for stable control of hypertension and prevention of cardiovascular diseases.
With the development of the Internet of Things technology, various physiological parameter monitoring devices based on wearable sensors are gradually becoming a research focus. However, there are still challenges in applying wearable device technology to wearable devices for commercial use and realtime and accurate blood pressure estimation. The main factors affecting daily physiological monitoring are environmental interference, electromagnetic interference and motion artifacts [3]. Environmental interference such as ambient light and electromagnetic interference can be passed through simple Bandpass filtering is removed, but in general, the frequency band of the motion artifact signal overlaps the frequency band of the patient's pulse wave measured by the received light sensor [4], so it cannot be removed by simple filtering.

Motion artifacts are caused by many different physical processes because of the motion of the subject. For example, the motion of the measurement system physically interferes with 
the signal path, which can be converted to motion artifacts [5]. Voluntary or involuntary movement of the subjects will lead to changes in the physiological state of the subjects, which may cause measurement errors due to inconsistent changes in external force and average pressure. These processes may occur individually or simultaneously, with the main processes depending on the measured modes and specific subject motions [6]. Motion artifacts are an important factor that limits the accuracy and universal applicability of wearable non-invasive blood pressure measurement.

\section{Motion Artifact Removal Methods}

For the application of wearable devices, the research on the removal of motion artifacts is particularly important. At present, there is no universal method that can solve all the motion artifact problems. At present, the research on motion artifact removal mainly focuses on two aspects of sensor system design and signal processing algorithm.

Wearable sensor is an important part of a wearable device, wearable sensor structure and system design is an important factor affecting motion artifacts, predecessors have done a lot of research on removing motion artifacts by sensor structure and system design. Sokwoo Rhee [7] et al. designed a miniature telemetry photoplethysmograph (PPG) sensor for long-term continuous monitoring, and proposed an efficient double ring design, which reduces the influence of external forces and acceleration on the sensor unit and reduces motion artifacts.

At present signal processing algorithms have powerful functions in removing motion artifacts. Various processing methods including adaptive filtering, independent component analysis and machine learning have been applied.

Adaptive filtering has the function of automatically adjusting its parameters and has been widely used in the field of nonlinear filtering. Generally, adaptive filtering requires additional motion tracking sensors to collect signals as the reference input of the adaptive filter. The MIT [8] used the MEMS accelerometer as a reference channel to reconstruct the signal obtained by their PPG sensor. Yalan Ye [9] et al. proposed a hybrid method combining adaptive filtering and signal decomposition, using a synchronized acceleration signal as a reference signal, and using a nonlinear adaptive filter based on second-order recursive least squares (RLS) to realizes denoising.

Independent component analysis (ICA) is a classic algorithm for blind source separation. Its application premise is that the pulse wave signal and the motion artifact signal are independent and uncorrelated, but the motion artifact and the pulse wave signal are not completely independent [9], so they are independent Component analysis has certain limitations. Milanesi [11] et al. used the frequency domain method together with the CICA (Temporally Constrained ICA) algorithm to remove motion artifacts in the Electrocardiography (ECG) and exemplified that this method provides better results compared to the basic ICA.

In addition, with the rapid development of artificial intelligence, signal processing algorithms based on machine learning have become one of the research hotspots, which brings new solutions to biomedical signal processing, especially the elimination of motion artifacts. Yunsik Son [12] et al. proposed a pulse waveform signal artifact removal model based on a deep belief network (DBN), which includes pulse segmentation, pressure normalization and denoising models using DBN, and applied the model to artifact removal in arterial blood pressure monitoring. Choon- Hian Goh [13] et al. designed a 1-D-CNN (Convolution Neural Network) to automatically learn the intrinsic features of the PPG waveform and perform the required classification and demonstrated the feasibility and effectiveness of applying blind signal processing and deep learning technology to PPG motion artifact detection.

\section{Conclusion}

This article reviews motion artifact removal technology of wearable blood pressure monitoring technology through the basic understanding and origin of motion artifacts. Although various algorithms have been developed to remove unwanted artifacts, the removal of motion artifacts is still an open research problem. There is currently no best method that can effectively and robustly remove motion artifacts under a wide range of conditions. As hardware and signal processing technologies continue to improve in terms of computing resources and costs, artifact removal systems will be easier to implement in non-clinical environments, increasing user comfort and reducing deployment costs.

\section{Acknowledgements}

This work was supported by the National Natural Science Foundation of China [No. 51875506], the Science Fund for Creative Research Groups of the National Natural Science Foundation of China [No.51821093].

\section{References}

1. The'odore H Macdonald (2006) Book Review: Preventing chronic diseases: a vital investment. RSPH 126(2): 95-95.

2. Jiao Xuejun, Fang Xingye (2002) Research on continuous measurement of blood pressure via characteristic parameters of pulse wave. Journal of biomedical engineering 19(2): 217-220.

3. KT Sweeney, TE Ward, S. McLoone (2012) Artifact Removal in Physiological Signals-Practices and Possibilities. IEEE Trans Inf Technol Biomed 16(3): 488-500.

4. M Raghuram, KV Madhav, EH Krishna, NR Komalla, K Sivani, et al. (2012) HHT based signal decomposition for reduction of motion artifacts in photoplethysmographic signals. I2MTC: 1730-1734. 
5. Seok Dongyeol, Lee Sanghyun, Kim Minjae, Cho Jaeouk, Kim Chul, et al. (2021) Motion artifact removal techniques for wearable EEG and PPG sensor systems. Front.Electron 2: 685513.

6. Hayes M J, Smith PR (2001) A new method for pulse oximetry possessing inherent insensitivity to artifact. IEEE Trans Biomed Eng 48(4): 452461.

7. Rhee S, Yang B H, Asada HH (2001) Artifact-resistant power-efficient design of finger-ring plethysmographic sensors. IEEE Trans Biomed Eng 48(7): 795-805.

8. Wood Levi B, Asada H Harry (2006) Noise cancellation model validation for reduced motion artifact wearable PPG sensors using MEMS accelerometers. Conf Proc IEEE Eng Med Biol Soc: 3525-3528.

9. Y Ye, Y Cheng, W He, M Hou, Z Zhang (2016) Combining nonlinear adaptive filtering and signal decomposition for motion artifact removal in wearable photoplethysmography. IEEE Sensors Journal 16(19): 71337141.
10. Fulai Peng, Zhengbo Zhang, Xiaoming Gou, Hongyun Liu, Weidong Wang (2014) Motion artifact removal from photoplethysmographic signals by combining temporally constrained independent component analysis and adaptive filter. BMC 13(1): 50.

11. M Milanesi, N Martini, N Vanello, V Positano, MF Santarelli, et al. (2008) Independent component analysis applied to the removal of motion artifacts from electrocardiographic signals. Med Biol Eng Comput 46(3): 251-261.

12. Yunsik Son, Seung-Bo Lee, Hakseung Kim, Eun-Suk Song, Hyub Huh, et al. (2018) Automated artifact elimination of physiological signals using a deep belief network: An application for continuously measured arterial blood pressure waveforms. Information Sciences 456: 145-158.

13. Choon-Hian Goh, Li Kuo Tan, Nigel H Lovell, Siew-Cheok Ng, Maw Pin Tan, et al. (2020) Robust PPG motion artifact detection using a 1-D convolution neural network. Comput Methods Programs Biomed 196: 105596. 